\title{
The three-dimensional matching problem in Kalmanson matrices
}

\author{
Sergey Polyakovskiy • Frits C.R. Spieksma • \\ Gerhard J. Woeginger
}

Published online: 16 November 2011

(C) The Author(s) 2011. This article is published with open access at Springerlink.com

\begin{abstract}
We investigate the computational complexity of several special cases of the three-dimensional matching problem where the costs are decomposable and determined by a so-called Kalmanson matrix. For the minimization version we develop an efficient polynomial time algorithm that is based on dynamic programming. For the maximization version, we show that there is a universally optimal matching (whose structure is independent of the particular Kalmanson matrix).
\end{abstract}

Keywords Computational complexity · Combinatorial optimization · Tractable case

\section{Introduction}

An instance of the classical three-dimensional matching problem (3DM) consists of $3 m$ points $1,2, \ldots, 3 m$ together with a cost function $c$ that assigns to every three-element subset $\{i, j, k\}$ of the points a corresponding cost $c(i, j, k)$. A feasible solution consists of $m$ pairwise disjoint three-element subsets of the points (that form a partition of the $3 m$ points); these three-element subsets are often called triples. The objective is to find a feasible solution that optimizes the total cost of all triples in the solution. 3DM is well-known to be NP-hard (Karp 1972; Garey and Johnson 1979).

\footnotetext{
S. Polyakovskiy $\cdot$ F.C.R. Spieksma

Operations Research Group, Katholieke Universiteit Leuven, Naamsestraat 69, 3000 Leuven, Belgium

S. Polyakovskiy

e-mail: sergey.polyakovskiy@econ.kuleuven.be

F.C.R. Spieksma

e-mail: frits.spieksma@econ.kuleuven.be

G.J. Woeginger $(\bowtie)$

Department of Mathematics, TU Eindhoven, P.O. Box 513, 5600 MB Eindhoven, The Netherlands e-mail: gwoegi@win.tue.nl
} 
Crama and Spieksma (1992) investigate a special case of 3DM where the cost coefficients are decomposable. This special case is built around an underlying symmetric distance function that assigns to every pair $i$ and $j$ of points a corresponding distance $d_{i j}$. The costs are then defined as

$$
c(i, j, k)=d_{i j}+d_{i k}+d_{j k} .
$$

3DM with decomposable costs is NP-hard, even if the distances $d_{i j}$ are the Euclidean distances of a set of points in the Euclidean plane (Spieksma and Woeginger 1996). In this paper, we are interested in decomposable costs where the distances $d_{i j}$ form a Kalmanson matrix.

Kalmanson matrices An $n \times n$ symmetric matrix $D=\left(d_{i j}\right)$ is a Kalmanson matrix if its entries fulfill the following two families of conditions:

$$
\begin{aligned}
& d_{i j}+d_{k \ell} \leq d_{i k}+d_{j \ell} \quad \text { for all } 1 \leq i<j<k<\ell \leq n, \\
& d_{i \ell}+d_{j k} \leq d_{i k}+d_{j \ell} \text { for all } 1 \leq i<j<k<\ell \leq n \text {. }
\end{aligned}
$$

Kalmanson matrices were introduced by Kalmanson (1975) in his investigations of special cases of the travelling salesman problem. They form a common generalization of the following three well-known families of distance matrices:

- The distance matrix of every convex point set in the Euclidean plane forms a Kalmanson matrix, if the points are numbered (say) in clockwise direction along the convex hull. The inequalities (2) and (3) state that in a convex quadrangle, the total length of two opposing sides is at most the total length of the two diagonals.

- Tree metrics correspond to Kalmanson matrices. Consider a rooted ordered tree with non-negative edge lengths, and number its leaves from left to right. Then the shortest path distances $d_{i j}$ between leaves $i$ and $j$ determine a Kalmanson matrix. The inequalities (2) and (3) are easily verified for trees with four leaves.

- Finally, for every interval $[\alpha, \beta]$ the corresponding cut matrix forms a Kalmanson matrix. The distance between points $i$ and $j$ is 0 , if both points lie in the interval or if both points lie outside the interval; the distance is 1 , if exactly one of $i, j$ lies in the interval.

Kalmanson matrices play a prominent role in combinatorial optimization. Christopher et al. (1996) (and independently Chepoi and Fichet 1998) showed that the Kalmanson conditions are equivalent to so-called circular decomposable metrics. Deineko et al. (1998) proved that the Kalmanson conditions are satisfied if and only if the distances have the so-called master tour property. Klinz and Woeginger (1999) analyzed the Steiner tree problem in Kalmanson matrices, and Deineko and Woeginger (1998) investigated a special case of the quadratic assignment problem where one of the matrices is a Kalmanson matrix.

Our results We investigate the three-dimensional matching problem with decomposable costs of the form (1) where the underlying distances $d_{i j}$ form a Kalmanson matrix. We consider the minimization version as well as the maximization version. For the minimization version, Sect. 2 develops a fast polynomial time algorithm that is based on dynamic programming. For the maximization version, Sect. 2 shows 

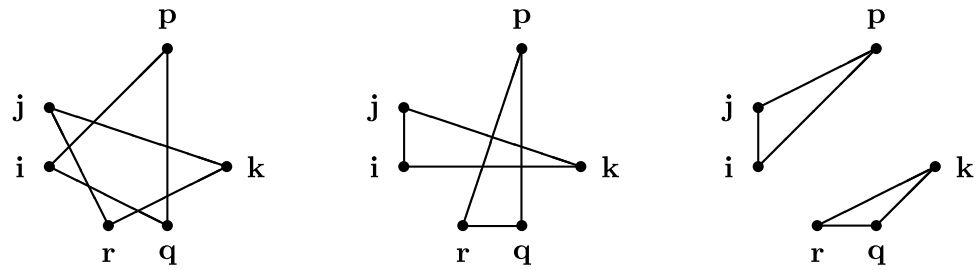

Fig. 1 Two interleaving triples (left); two overlapping triples (middle); two separated triples (right)

that there is a universally optimal matching (whose structure does not depend on the particular underlying Kalmanson matrix). In Sect. 3 we discuss higher-dimensional matching variants with appropriately defined decomposable cost coefficients arising from Kalmanson matrices. The maximization version is also easy in the higherdimensional case, but the complexity of the minimization version remain unclear.

\section{Three-dimensional matchings}

In this section we consider the three-dimensional matching problem in Kalmanson matrices. We show that the minimization version is solvable in polynomial time by dynamic programming, and that the maximization version has a universally optimal matching.

If the points in a Kalmanson matrix are cyclically shifted and renumbered, then all inequalities in (2) and (3) remain valid. Hence, from now on we will assume that these points are actually arranged in a cyclic order. Then with respect to this cyclic order, two point triples can either be interleaving, or overlapping, or separated; see Fig. 1 for an illustration. Note that there are three partitions into separated triples, six partitions into overlapping triples, and a unique partition into interleaving triples. The following lemma shows that interleaving triples form the most expensive solution, and that the cheapest solution can be found among the partitions into separated triples.

Lemma 2.1 Consider six cyclically ordered points whose distances satisfy the Kalmanson inequalities. Consider a partition of the six points into two overlapping triples $t_{1}$ and $t_{2}$.

(a) There exists a partition into separated triples whose objective value is at most the objective value of $t_{1}$ and $t_{2}$.

(b) The objective value of $t_{1}$ and $t_{2}$ is at most the objective value of the partition into interleaving triples.

Proof Assume that the cyclic order of the six points is $i<j<p<k<q<r$, and that $t_{1}=(i, j, k)$ and $t_{2}=(p, q, r)$; see the middle picture in Fig. 1 for an illustration. Inequality (2) with $j<p<k<r$ yields

$$
d_{j p}+d_{k r} \leq d_{j k}+d_{p r}
$$

and inequality (2) with $i<p<k<q$ yields

$$
d_{i p}+d_{k q} \leq d_{i k}+d_{p q} .
$$


By summing these two inequalities and by adding $d_{i j}+d_{q r}$ to both sides, we get

$$
\left(d_{i j}+d_{j p}+d_{i p}\right)+\left(d_{q r}+d_{k r}+d_{k q}\right) \leq\left(d_{i j}+d_{j k}+d_{i k}\right)+\left(d_{q r}+d_{p r}+d_{p q}\right) .
$$

This proves (a), since the triples $(i, j, p)$ and $(q, r, k)$ are separated. In a similar fashion we derive

$$
d_{i j}+d_{p r} \leq d_{i p}+d_{j r}
$$

from $i<j<p<r$, and

$$
d_{i k}+d_{q r} \leq d_{i q}+d_{k r}
$$

from $i<k<q<r$, respectively. By summing and then adding $d_{j k}+d_{p q}$ to both sides we get the inequality

$$
\left(d_{i j}+d_{j k}+d_{i k}\right)+\left(d_{q r}+d_{p r}+d_{p q}\right) \leq\left(d_{i p}+d_{i q}+d_{p q}\right)+\left(d_{j r}+d_{k r}+d_{j k}\right) \text {. }
$$

This proves $(\mathrm{b})$, since the triples $(i, p, q)$ and $(r, j, k)$ are interleaving.

Lemma 2.2 Let $D=\left(d_{i j}\right)$ be a $3 m \times 3 m$ Kalmanson matrix.

(a) There exists an optimal solution for the minimization version of $3 D M$ in $D$ in which every pair of triples is separated.

(b) There exists an optimal solution for the maximization version of $3 D M$ in $D$ in which every pair of triples is interleaving.

Proof We introduce an auxiliary Kalmanson matrix $D^{\prime}=\left(d_{i j}^{\prime}\right)$ that satisfies all inequalities in (2) and (3) with strict inequality. The objective value of a threedimensional matching with respect to matrix $D^{\prime}$ is called its pseudo-cost.

For (a), we select among all optimal three-dimensional matchings (that minimize the cost with respect to matrix $D$ ) a matching $M$ that has the smallest pseudo-cost. We claim that in the optimal solution $M$ every pair of triples is separated. Suppose not. Then there are two triples $t_{1}$ and $t_{2}$ that interleave or overlap. By Lemma 2.1, these two triples can be replaced by two other triples that are separated. This either decreases the objective value (and hence yields a contradiction to the optimality of $M$ ), or it yields another optimal matching with strictly better pseudo-cost (which again is a contradiction).

For (b), we select among all optimal three-dimensional matchings (that maximize the cost with respect to $D$ ) a matching $M$ that has the largest pseudo-cost. Similarly as in the proof of (a), one sees that in $M$ every pair of triples is interleaving.

Lemma 2.2(a) suggests a dynamic programming approach for the minimization version. For two points $a$ and $b$, we denote by $P[a, b]$ the set of points $a, a+1, \ldots, b-2, b-1$ in the cyclic order (note that for $a \geq b$, the set $P[a, b]$ contains the points $a, \ldots, 3 m$ and the points $1, \ldots, b-1)$. By $V[a, b]$ we denote the smallest possible objective value of a three-dimensional matching for $P[a, b]$ : If the cardinality of $P[a, b]$ is a multiple of three then $V[a, b]$ exists and takes a finite value, and if the cardinality of $P[a, b]$ is not a multiple of three then we set $V[a, b]=\infty$.

We show how to compute all values $V[a, b]$ in $O\left(\mathrm{~m}^{4}\right)$ overall time. In an initialization phase, we consider the trivial cases with $|P[a, b]|=3$ and compute the 
corresponding values $V[a, b]$. The remaining values are handled in order of increasing cardinality of $P[a, b]$. If $|P[a, b]|$ is a multiple of three, we proceed as follows. By Lemma 2.2 we only need to consider matchings in which every pair of triples is separated. The shortest such matching for $P[a, b]$ consists of a triple $(a, x, y)$ (where $x$ precedes $y$ in the cyclic ordering of $P[a, b]$ ), a shortest matching for the remaining points between $a$ and $x$, a shortest matching for the remaining points between $x$ and $y$, and a shortest matching for the remaining points between $y$ and $b-1$. The corresponding objective value is

$$
\left(d_{a x}+d_{a y}+d_{x y}\right)+V[a+1, x]+V[x+1, y]+V[y+1, b] .
$$

We compute $V[a, b]$ as the minimum of all such values for $x, y \in P[a, b]$ with $a<x<y$ in the cyclic ordering. There are $O\left(\mathrm{~m}^{2}\right)$ values to compute, and the computation of every single value takes $O\left(\mathrm{~m}^{2}\right)$ time; this gives an overall time complexity of $O\left(m^{4}\right)$.

In the end, the optimal objective value can be found in $V[1,1]$. By storing appropriate auxiliary information in the states of the dynamic program, one can also compute the corresponding optimal matching within the same time complexity. These are standard techniques, and we do not elaborate on them. All in all, this yields the following theorem.

Theorem 2.3 The minimization version of 3DM in Kalmanson matrices can be solved in polynomial time $O\left(\mathrm{~m}^{3}\right)$.

Next, let us turn to the maximization version of three-dimensional matching in Kalmanson matrices. It turns out that this problem has a universally optimal solution, and hence can be solved in linear time $O(m)$ without even looking at the Kalmanson matrix.

Theorem 2.4 The maximization version of $3 D M$ in a $3 m \times 3 m$ Kalmanson matrix is always solved to optimality by the following matching: For $\ell=1, \ldots, m$ use the triple $(\ell, \ell+m, \ell+2 m)$.

Proof By Lemma 2.2(b), there exists an optimal matching $M$ in which every pair of triples is interleaving. We claim that $M$ is of the desired form.

Consider a triple $t_{1}=(i, j, k) \in M$ with $i<j<k$. If $j>i+m$, then there are at least $m$ points $i+1, i+2, \ldots, j-1$ between $i$ and $j$, two of which must belong to the same triple $t_{2}$ in $M$. Then $t_{1}$ and $t_{2}$ are not interleaving, and we have a contradiction. The cases where $k>j+m$ and $k<i+2 m$ lead to similar contradictions.

\section{Higher-dimensional matchings}

In this section we investigate $k$-dimensional matching problems, where a set of $n=$ $\mathrm{km}$ points $1,2, \ldots, \mathrm{km}$ has to be divided into $m$ subsets $T_{1}, \ldots, T_{m}$ of cardinality $k$; such a $k$-element subset will also be called a $k$-cluster or simply a cluster, for short. 
The cost of a $k$-cluster $T$ depends on an underlying symmetric Kalmanson matrix $D=\left(d_{i j}\right)$, and is given by

$$
c(T)=\sum_{i \in T} \sum_{j \in T: j>i} d_{i j} .
$$

The goal is to find a $k$-dimensional matching that minimizes or maximizes the total cost of all $m$ clusters. We say that two clusters $T_{1}$ and $T_{2}$ are separated, if there exists an interval (in the cyclic ordering) that contains all points of one cluster, but no point of the other cluster. We say that $T_{1}$ and $T_{2}$ are interleaving, if between any two consecutive points of $T_{1}$ (in the cyclic ordering) there lies exactly one point of $T_{2}$. Note that for $k=3$ these definitions coincide with the corresponding definitions for separated and interleaving triples in the preceding section, and that for $k=3$ the costs in (4) boil down to the decomposable costs in (1).

We will first discuss the maximization version of this $k$-dimensional matching problem, and we will generalize Theorem 2.4 to the higher-dimensional case. For this we use the structural characterization of Kalmanson matrices in Proposition 3.1 below, which is based on the following definitions. A matrix $S=\left(s_{i j}\right)$ is called a sum matrix if there are real numbers $\sigma_{1}, \ldots, \sigma_{n}$ such that $s_{i j}=\sigma_{i}+\sigma_{j}$ for all $1 \leq i, j \leq n$. For integers $\alpha$ and $\beta$ with $1 \leq \alpha \leq \beta \leq n$, define the cut matrix $C^{[\alpha, \beta]}$ by its entries

$$
c_{i j}^{[\alpha, \beta]}= \begin{cases}1 & \text { if } i \in[\alpha, \beta] \text { and } j \notin[\alpha, \beta], \text { or } i \notin[\alpha, \beta] \text { and } j \in[\alpha, \beta] \\ 0 & \text { otherwise. }\end{cases}
$$

In other words, a cut matrix $C^{[\alpha, \beta]}$ encodes the cut between the interval $[\alpha, \beta]$ and the complement of this interval. It is well-known (and easily verified) that all sum matrices and all cut matrices are Kalmanson matrices. Note that the sum of two Kalmanson matrices is again a Kalmanson matrix, and note that multiplying a Kalmanson matrix by a non-negative real number yields another Kalmanson matrix. Hence the Kalmanson matrices form a cone. The extreme rays of this Kalmanson cone are characterized in the following proposition.

Proposition 3.1 (Bandelt and Dress 1992; Christopher et al. 1996; Chepoi and Fichet 1998) A matrix $D$ is a Kalmanson matrix if and only if there exist non-negative real numbers $x_{\alpha, \beta}$ for $1 \leq \alpha \leq \beta \leq n$ and a sum matrix $S$, such that

$$
D=S+\sum_{\alpha=1}^{n} \sum_{\beta=\alpha}^{n} x_{\alpha, \beta} C^{[\alpha, \beta]} \text {. }
$$

The following lemmas investigate the extreme rays of the Kalmanson cone with respect to $k$-dimensional matchings. They all deal with instances where $2 k$ points have to be divided into two $k$-clusters.

Lemma 3.2 Let $S=\left(s_{i j}\right)$ be a $2 k \times 2 k$ sum matrix with $s_{i j}=\sigma_{i}+\sigma_{j}$. Then all partitions into two $k$-clusters yields the same objective value for the $k$-dimensional matching problem. 
Proof Consider an arbitrary partition into two $k$-clusters $T_{1}$ and $T_{2}$. The cost of cluster $T_{1}$ according to (4) can be rewritten as

$$
c\left(T_{1}\right)=\sum_{i \in T_{1}} \sum_{j \in T_{1}: j>i} \sigma_{i}+\sigma_{j}=(k-1) \sum_{i \in T_{1}} \sigma_{i} .
$$

Hence the total cost $c\left(T_{1}\right)+c\left(T_{2}\right)=(k-1) \sum_{i=1}^{2 k} \sigma_{i}$ is indeed independent of the concrete partition.

Lemma 3.3 Let $C^{[\alpha, \beta]}$ be a $2 k \times 2 k$ cut matrix for the interval $[\alpha, \beta]$ with $1 \leq \alpha \leq$ $\beta \leq 2 k$. Then the (unique) partition into two interleaved $k$-clusters yields the maximimum objective value.

Proof Consider an arbitrary partition into two clusters $T_{1}$ and $T_{2}$. Let $\ell=\beta-\alpha+1$ denote the number of points in interval $[\alpha, \beta]$, and let $x$ denote the number of points from interval $[\alpha, \beta]$ in $T_{1}$. Note that in case $T_{1}$ and $T_{2}$ are interleaved, then $x=\lfloor\ell / 2\rfloor$ or $x=\lceil\ell / 2\rceil$. Since $T_{1}$ contains $k-x$ points not in $[\alpha, \beta]$, and since $T_{2}$ consists of $\ell-$ $x$ points in the interval and of $k-\ell+x$ points outside the interval, the corresponding objective value is

$$
c\left(T_{1}\right)+c\left(T_{2}\right)=2 x(k-x)+2(\ell-x)(k-\ell+x)=2 \ell(k-\ell)+4 \ell x-4 x^{2} .
$$

This function is concave, and takes its maximum over the integers at $x=\lfloor\ell / 2\rfloor$ and at $x=\lceil\ell / 2\rceil$. Hence interleaved clusters indeed are maximizers.

Lemma 3.4 Let $D$ be a $2 k \times 2 k$ Kalmanson matrix. Then the (unique) partition into two interleaved $k$-clusters yields the maximimum objective value.

Proof By Proposition 3.1, matrix $D$ can be written as a non-negative linear combination of cut matrices and a sum matrix. By Lemmas 3.2 and 3.3 the partition into two interleaved clusters is optimal for every cut matrix and every sum matrix, and therefore also for matrix $D$.

With the help of Lemma 3.4, we can easily raise the proofs of Lemma 2.2(b) and Theorem 2.4 from the case $k=3$ to the general $k$-dimensional case with $k \geq 3$ : There always exists an optimal solution for the maximization version of $k$-dimensional matching in which every pair of $k$-clusters is interleaving, which leads to the following result.

Theorem 3.5 The maximization version of $k$-dimensional matching in a $\mathrm{km} \times \mathrm{km}$ Kalmanson matrix is always solved to optimality by the following partition: For $\ell=$ $1, \ldots, m$ the cluster $T_{\ell}$ consists of the $k$ points $\ell, \ell+m, \ell+2 m, \ldots, \ell+(k-1) m$.

We do not understand the complexity of the minimization version of $k$-dimensional matchings for $k \geq 4$. The approach for $k=3$ from the preceding section does not carry over to $k \geq 4$ : The following example demonstrates that the tools developed for $k=3$ in Lemma 2.1 and Lemma 2.2 break down for $k \geq 4$. 
Fig. 2 The tree $T_{k}$ in Example 3.6 has $2 k$ leaves

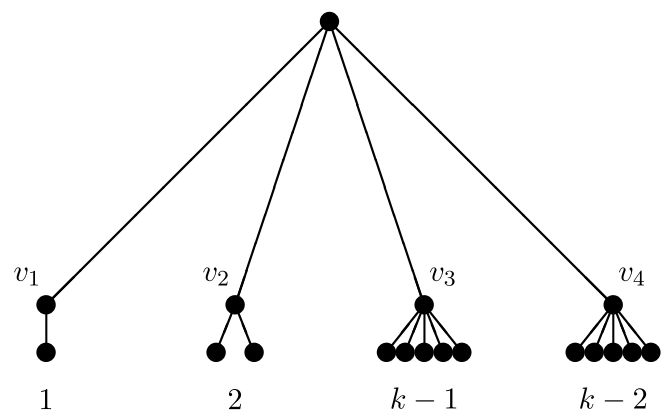

Example 3.6 For $k \geq 4$, consider the edge-weighted rooted tree $T_{k}$ on $2 k+5$ vertices. The root $r$ has four children $v_{1}, v_{2}, v_{3}, v_{4}$. Vertex $v_{1}$ has a single child, vertex $v_{2}$ has two children, vertex $v_{3}$ has $k-1$ children, and vertex $v_{4}$ has $k-2$ children. All four edges between $r$ and $v_{1}, v_{2}, v_{3}, v_{4}$ have length 1 , and all edges between $v_{1}, v_{2}, v_{3}, v_{4}$ and the $2 k$ leaves have length $\varepsilon$, where $\varepsilon>0$ is some tiny real number; see Fig. 2 for an illustration.

If we number the leaves in $T_{k}$ from left to right, the shortest path distances $d_{i j}$ between leaf $i$ and leaf $j$ yield a Kalmanson matrix. The corresponding instance of $k$-dimensional matching asks for a partition into two $k$-clusters. The unique optimal solution matches the single child of $v_{1}$ with the $k-1$ children of $v_{3}$, and it matches the two children of $v_{2}$ with the $k-2$ children of $v_{4}$. The resulting two clusters are not separated.

\section{Conclusions}

We have derived a number of results on the minimization version and on the maximization version of $k$-dimensional matchings in Kalmanson matrices. The maximization version turned out to be easy for all $k \geq 3$, and the structure of the optimal solution does not even depend on the data. The minimization version is polynomial time solvable for $k=3$, but our approach is based on combinatorial structures that disappear in the cases with $k \geq 4$.

Pinpointing the computational complexity of the minimization version for $k=4$ is a challenging open problem. We suspect that there is a polynomial time algorithm for it. In any case, new approaches with substantially different ideas are needed to make progress on this problem.

Open Access This article is distributed under the terms of the Creative Commons Attribution Noncommercial License which permits any noncommercial use, distribution, and reproduction in any medium, provided the original author(s) and source are credited.

\section{References}

Bandelt HJ, Dress AWM (1992) A canonical decomposition theory for metrics of a finite set. Adv Math 92:47-105 
Chepoi V, Fichet B (1998) A note on circular decomposable metrics. Geom Dedic 69:237-240

Christopher G, Farach M, Trick M (1996). The structure of circular decomposable metrics. In: Proceedings of the 4th annual European symposium on algorithms (ESA'1996). LNCS, vol 1136. Springer, Berlin, pp 486-500

Crama Y, Spieksma FCR (1992) Approximation algorithms for three-dimensional assignment problems with triangle inequalities. Eur J Oper Res 60:273-279

Deineko VG, Rudolf R, Woeginger GJ (1998) Sometimes travelling is easy: the master tour problem. SIAM J Discrete Math 11:81-93

Deineko VG, Woeginger GJ (1998) A solvable case of the quadratic assignment problem. Oper Res Lett 22:13-17

Garey MR, Johnson DS (1979) Computers and intractability: a guide to the theory of NP-completeness. Freeman, San Francisco

Kalmanson K (1975) Edgeconvex circuits and the travelling salesman problem. Can J Math 27:1000-1010

Karp RM (1972) Reducibility among combinatorial problems. In: Miller RE, Thatcher JW (eds) Proceedings of the complexity of computer computations. Plenum Press, New York, pp 85-104

Klinz B, Woeginger GJ (1999) The Steiner tree problem in Kalmanson matrices and in Circulant matrices. J Comb Optim 3:51-58

Spieksma FCR, Woeginger GJ (1996) Geometric three-dimensional assignment problems. Eur J Oper Res 91:611-618 